\title{
Análisis de la primarización de la economía en América Latina desde la base material del método dialéctico
}

Analysis of the primarization of the economy in Latin America from the material basis of the dialectical method

Renato Sánchez Proaño. ${ }^{1}$

\begin{abstract}
.
DOI: https://doi.org/10.33262/concienciadigital.v4i3.2.1852

In recent years, Latin America has not only experienced economic growth, but has also had to face a trend toward primarization of the economy, deindustrialization, and the increase in assembly plants and assembly plants. A classic geopolitical analysis would reveal that the problem lies in the inability of their rulers to manage the states, whatever their political position. Ethical reasoning like these is not enough to achieve a pragmatic change in reality; reason for which, the present work tries to realize the analysis from the method of the historical materialism and the ecological economy. For this, in the first place, the historical and epistemological character of capitalism is revealed, with which categories can be identified that go beyond the selfish tendency of "homo economicus". Second, the conceptual similarities between dialectical materialism and ecological economics have been expressed, which incorporates new epistemes that recognize the value that nature has for life, something that neoclassical environmental economics has

\footnotetext{
${ }^{1}$ Universidad Politécnica Salesiana. Grupo de Investigación en Ecología y Gestión de los Recursos Naturales, Pichincha, Quito, Ecuador. rsanchezp@ups.edu.ec. Universidad Andina Simón Bolívar. Candidato a Dr. en Salud Colectiva, ambiente y sociedad, Quito, Ecuador. https://orcid.org/0000-00025015-7570.
} 
not achieved. For this, techniques of analysis of society-nature metabolism are used, which shows how Latin America's participation in the world economy has been as a supplier of raw materials since colonial times; and how, in turn, nature has dialectically determined the region materially at its economic and social levels; continuing and accentuating this trend even during times of economic growth.

Keywords: Metabolism, society-nature, ecological economy, capitalism.

\section{Resumen.}

En los últimos años América Latina no solo ha experimentado un crecimiento económico, sino también ha tenido que enfrentar una tendencia a la primarización de la economía, a la desindustrialización y al aumento de las maquiladoras y ensambladoras. Un análisis geopolítico clásico revelaría que el problema está en la incapacidad de sus gobernantes de gestionar los estados, sea cual sea el posicionamiento político que tengan. Razonamientos éticos como estos no son suficientes para lograr un cambio pragmático de la realidad; razón por la cual, el presente trabajo pretende realizar el análisis desde la base material del método dialéctico y la economía ecológica. Para esto, en primer lugar, se devela el carácter histórico y epistemológico del capitalismo, con lo cual se pueden identificar categorías que van más allá de la tendencia egoísta del "homo economicus". En segundo lugar, se han expresado las similitudes conceptuales entre la base material del método dialéctico y la economía ecológica, el cual incorpora nuevas epistemes que reconocen la importancia que la naturaleza tiene para la vida, algo que la economía ambiental basado en las teorías neoclásicas no ha logrado. Para esto se usan técnicas de análisis del metabolismo sociedad-naturaleza, el cual demuestra cómo desde la colonia la participación de América Latina en la economía mundial ha sido como proveedor de materias primas; y cómo a su vez la naturaleza dialécticamente ha determinado materialmente a la región en sus niveles económicos y sociales; continuándose y acentuándose esta tendencia incluso durante las épocas de crecimiento económico.

Palabras claves: Metabolismo sociedad-naturaleza, economía ecológica, capitalismo.

\section{Introducción.}

Visto desde la geopolítica ortodoxa, los recursos de América Latina son objetos en disputa para el dominio de los países centrales (Dallanegra 2010), y de resistencia para la población latinoamericana. Esto lleva al planteamiento de varios posicionamientos éticos en los cuales los estados y sus gobernantes son juzgados como los principales cómplices de la extracción de recursos, sin importar si estos tienen tendencias conservadoras o progresistas.

La realización de juzgamientos éticos y morales del mundo pueden ser el inicio de la develación de problemas de inequidad social, pero no son suficientes para un cambio pragmático de la realidad. Pararse desde una posición latinoamericanista y centrarse en 
el juzgamiento moral del mundo no cambia la realidad. De igual manera, quienes promulgan soluciones con tendencias neoliberales consideran que la distribución de la riqueza y los problemas ambientales los resolverá el mercado. Para esto afirman, se requiere una etapa inicial de desarrollo, la cual puede empezar según Rostow (1961) con la exportación de recursos naturales; creyéndose que la riqueza generada en esta etapa posteriormente será distribuida equitativamente. Este planteamiento desarrollado por Kuznets (1955) se lo explica en el Figura 1, donde el punto 1 indica un estado de pobreza y equidad social, que luego de desarrollarse una actividad productiva (como la extracción de recursos naturales para el caso de América Latina) aumenta la inequidad (punto 2), para luego reducirse por la distribución equitativa del marcado (punto 3).

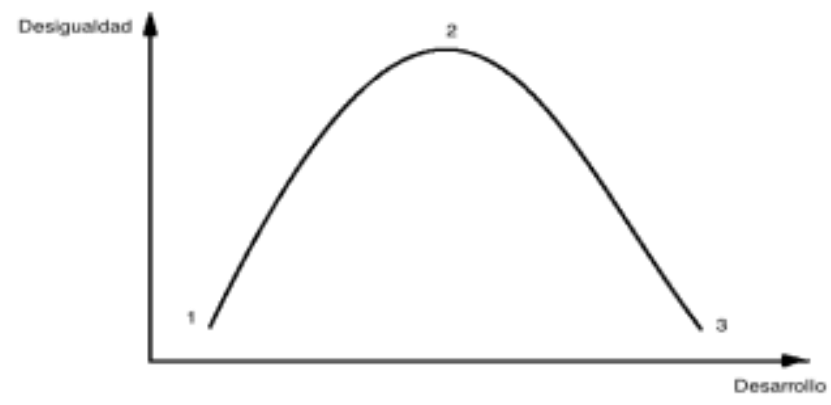

Figura 1. Curva de Kuznets

Fuente: Kuznets, 1955

Este paradigma es compartido por organizaciones que rigen la economía mundial en la actualidad como son el Banco Mundial o el Fondo Monetario Internacional (Banco Mundial, 2010); a pesar de existir evidencias notorias del aumento de la inequidad y una nula estabilización de la curva del Gráfico 1 (WIDER, 2000).

La búsqueda de una explicación inmediata desde la ciencia geopolítica hegemónica, en la cual las regiones del mundo están repartidas en áreas territoriales de influencia de las grandes transnacionales (Haushofer, 1986) tampoco permite captar la unidad histórica a el cual este fenómeno pertenece; ya que, donde se encuentran los países no depende únicamente de su posición en el mapa, sino también de un pasado que carga con contingencias de interacción a sus componentes tanto en el presente como en el futuro (Lewontin et al., 1984). Por lo que se plantean como objetivos del presente trabajo la realización de una crítica de la economía política a través de la base material del método dialéctico y la economía ecológica para analizar esta complejidad, y porqué la realidad se ha constituido como la conocemos.

\section{Metodologia.}

El diseño de la investigación presenta un enfoque crítico a nivel documental, apoyado en la base material del método dialéctico, la cual es una concepción del mundo desarrollada por Marx para la comprensión de la historia humana en su totalidad (Marx, 2002). En este método se trata de investigar a la sociedad humana desde su superestructura; para lo cual se parte de individuos empíricos y las relaciones que se establecen entre ellos (Marx, 1974), siendo la más importante sus formas de producción y reproducción. 
No se puede juzgar a un individuo por lo que piensa de sí mismo, como tampoco se puede juzgar a una época por su propia conciencia social (Marx, 1980), y esto se debe a que la conciencia de una sociedad en un momento dado no solo es fruto de una casualidad evolucionada (Eysenck, 1971), sino que también es determinada en un marco de saber acorde a una "verdad" impuesta desde un poder para una cierta época (Foucault, 1966). Difícilmente en este tiempo dominado por el sistema capitalista se pueda entender o concebir las cosas y las palabras fuera del marco epistemológico hegemónico.

Una metodología estará adscrita a una episteme (Guba, 1990); es por esto que el método propuesto en este trabajo pretende entender la primarización de la economía en América Latina no desde un modelo de conocimiento ligado a una episteme hegemónica; sino desde la apreciación ontológica. Estas categorías se las trata de organizar en el Gráfico 1.

Gráfico 1

Organización de la ontología, epistemología y metodología

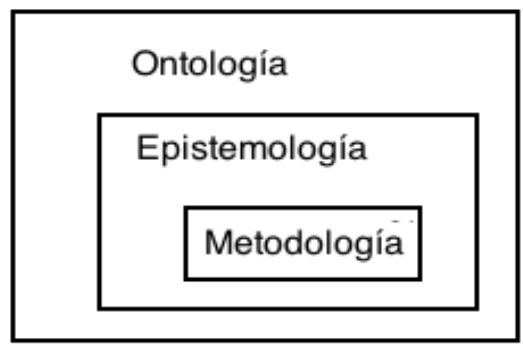

Nota: Una metodología está basada en una epistemología temporal y espacial, que se a su vez forma parte de una ontología.

Fuente: Breilh, 2011.

La complejidad del análisis materialista histórico hace que la compresión de una sociedad no se base únicamente en la suma de los factores característicos de sus individuos; por el contrario, pone en evidencia interacciones entre los individuos, grupos sociales o sociedades organizadas entre sí, junto con la naturaleza; y cómo a su vez la naturaleza también condiciona a estas distintas escalas humanas. Este es un método desarrollado por Breilh (2011), que se ejemplifica en el Gráfico 2 para estudios epidemiológicos, y que sirven como método para operativizar el estudio complejo de la sociedad en el presente trabajo.

Gráfico 2

Método de análisis de la complejidad

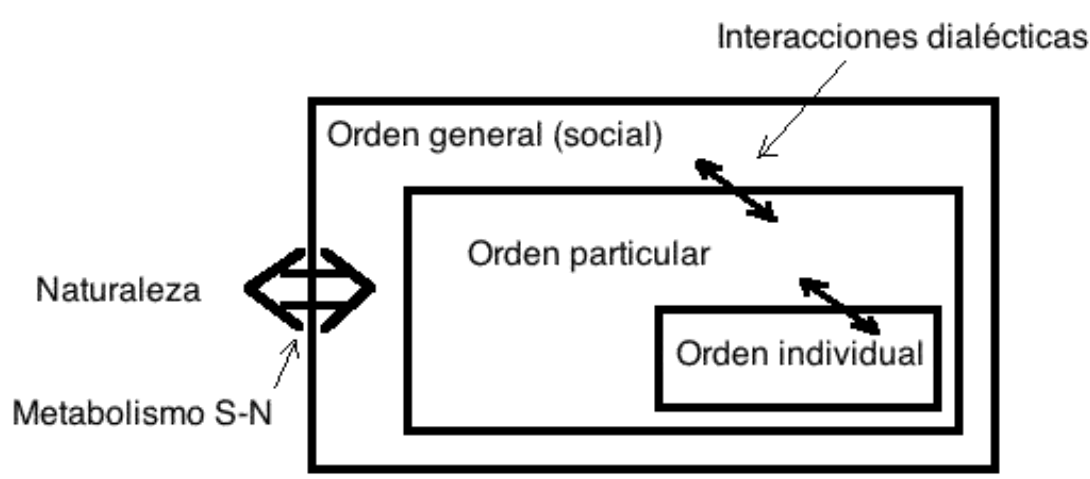

Fuente: Breilh, 2011. 
En este gráfico se puede observar como el individuo influencia y es influenciado por su entorno más cercano, el cual es conocido como orden particular. A su vez, el orden particular es influenciado e influencia a un orden social más amplio y complejo; y es este orden social general el que interactúa dialécticamente con la naturaleza, ya que la condiciona o es condicionado por esta.

\section{Resultados.}

\section{Historicidad del capitalismo}

El capitalismo se muestra como un sistema de origen histórico espontáneo y natural, justificado en que los seres humanos tienen una fuerte tendencia al egoísmo y a "realizar trueques, cambios e intercambios de unas cosas por otras" (Smith, 1994), para obtener una mayor utilidad individual (Gossen, 1881). Para Marx, lo único natural, espontaneo y por tanto ontológico es la necesidad de transformar la naturaleza para satisfacer las necesidades humanas, lo cual se ejecuta mediante el trabajo (Marx 1974); mientras que el capitalismo se presenta como una configuración histórica de ese proceso (Echeverría, 1998); y por tanto es ubicado como una categoría epistemológica, teniendo un inicio y posteriormente un fin.

El no ser críticos, y creer que el sistema capitalista es el único modelo que existirá de forma indefinida y en la manera en que lo conocemos, es un dogmatismo; ya que este sistema se ha desarrollado históricamente, existen grandes fuerzas para su conservación, y pueden existir otras grandes fuerzas sociales o naturales para su reemplazo. Para cambiar el enfoque epistemológico y llegar a un razonamiento apropiado, se requiere del razonamiento crítico; de esta forma se pueden identificar sin restricciones de visión cuales métodos funcionan y cuales no (Kant, 2005).

La historia moderna comienza en el Siglo XVI con la creación de un comercio y mercado expansivos. La historia moderna de América Latina se desarrolla paralelamente a la del capitalismo, asignándose a la región en la época de la conquista el papel de periferia exportadora de materias primas e importadora de bienes de lujo. Siglos después, problemas internos y externos al territorio causaron revoluciones impulsadas por los criollos, quienes una vez con el poder cambiaron el comercio monopólico de España por la de otras potencias. Estos pactos no reemplazaron los modelos económicos de América Latina, sino que la determinó a ser un proveedor de materias primas, y comprador de productos industriales, tendencia que continúa hasta estos días (Sánchez, 2017).

\section{La economía ambiental no es suficiente}

A lo largo de los años se ha demostrado que la naturaleza tiene límites, y que el aumento de la extracción de recursos naturales no puede realizarse de manera ilimitada (Meadows et al., 1972); es por lo que se ha creado una nueva rama desde las economías neoclásicas para el manejo ambiental, conocida como economía ambiental. Para Field et al. (2003: 3) la economía ambiental es "la aplicación de los principios económicos al estudio de la gestión de los recursos naturales”. En esta definición claramente se puede notar la 
creencia en que los principios económicos del capitalismo van más allá del tiempo y de las leyes físicas; y que la gestión de los recursos naturales se puede lograr mediante las leyes de las economías neoclásicas. Las deficiencias de estas técnicas han hecho necesaria la búsqueda de nuevas epistemes que conecten la naturaleza con las formas de producción sociales. Estas nuevas perspectivas se pueden estar gestando en nuevas ciencias como por ejemplo la economía ecológica, la cual parte de una noción distinta de la naturaleza, en la cual las leyes de la naturaleza son las que rigen la apropiación de los "recursos naturales" necesarios en la producción social y no viceversa (Martínez-Alier, 1999).

Para Martínez-Alier (2005), "la economía neoclásica" está limitada por la naturaleza; y, por tanto, las leyes sociales que rigen la generación de valor están circunscritas a las leyes físicas naturales, como trata de ejemplificar la economía ecológica. Esta concepción del sistema económico toma en cuenta la base material, y por tanto las leyes físicas y biológicas en la organización humana (Engels, 1961). Como lo hace la base material del método dialéctico. En el Gráfico 3 se muestra un esquema explicativo de las economías neoclásicas, notándose que esta reduce a la economía a un intercambio de bienes y servicios entre seres humanos, sin tomar en cuenta a la naturaleza.

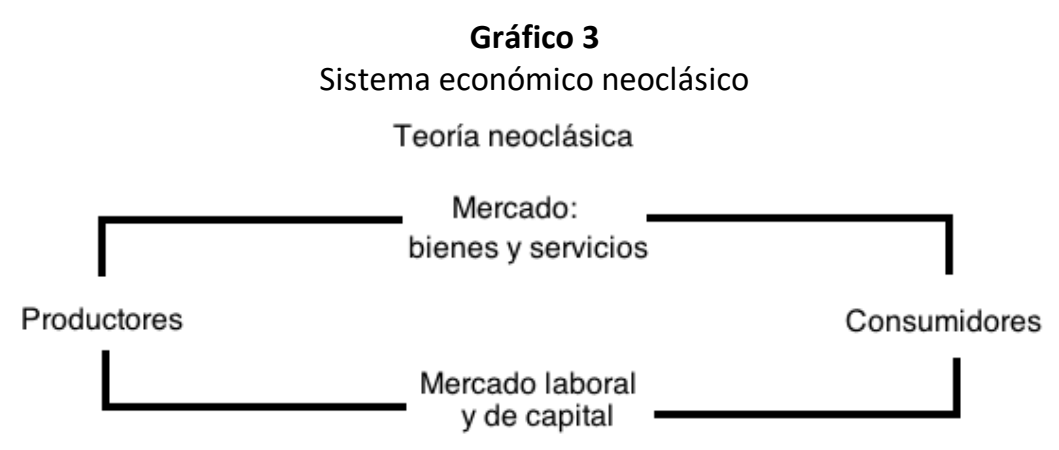

Fuente: Martínez-Alier, 2005

Contrario a esto, en el Gráfico 4 se muestra el sistema de la economía ecológica; en el cual el intercambio en la sociedad es solo una parte de los procesos que se dan en la naturaleza.

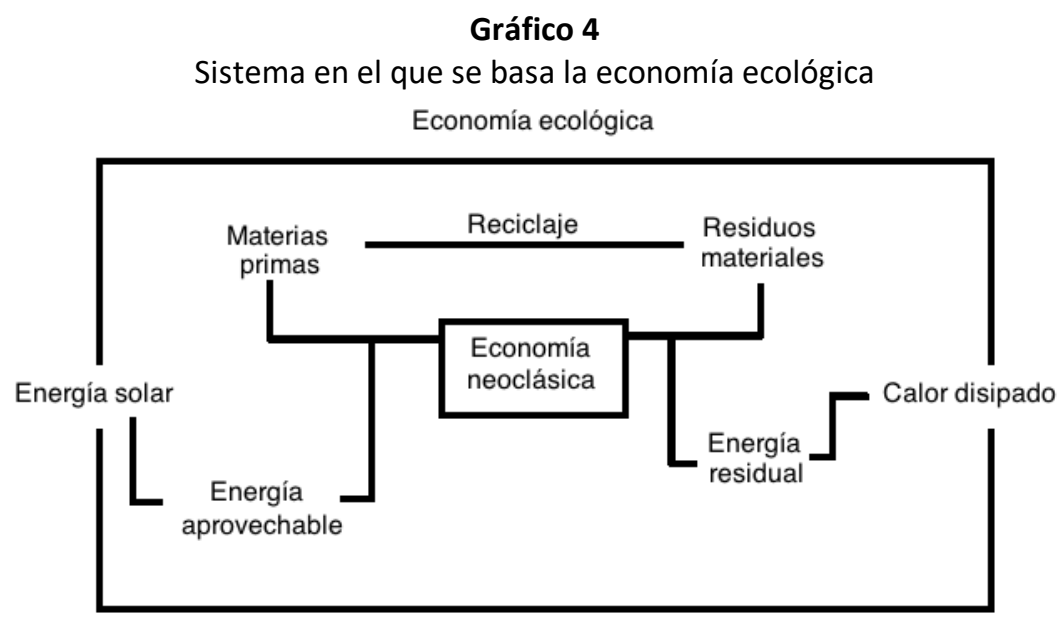

Fuente: Martínez-Alier, 2005. 
La base material del método dialéctico y la economía ecológica presentan varias similitudes y diferencias; dentro de sus similitudes está justamente la incorporación de las leyes de la naturaleza; mientras que su mayor diferencia está en el que la economía ecológica no cuestiona al régimen económico y social dominante (Torres 2015).

Dentro de la incorporación de las leyes de la naturaleza están la materialidad física y biológica, las cuales funcionan en todo momento, y sus leyes rigen y afectan a las actividades sociales y por tanto económicas. Entre la materialidad física y la materialidad biológica se presenta un gran salto cualitativo, que hace que las leyes biológicas no puedan ser explicadas únicamente con las leyes de la física. Una diferencia fundamental entre materialidad física y biológica es que esta última requiere la interacción entre organismos vivos y otros materiales en un ecosistema común para su funcionamiento (Tansley 1934).

El mayor salto cualitativo se da al pasar de materialidad biológica a materialidad social; ya que esta reúne los principios de la materialidad física y biológica de la cual forma parte, e incorpora a estas la subjetividad (Cornejo et al. 2016). El no solo satisfacer las necesidades físicas, sino también las subjetivas hace que el humano acumule en su historia el desarrollo de las posibilidades de transformación de la materia según sus capacidades y necesidades (Marx, 1971).

La inclusión de leyes físicas y biológicas en las ciencias sociales no significa que exista un solo tipo de análisis para todos los tipos de problemas, ya que no existe una forma de reducir la complejidad propia de la materialidad biológica o social a la materialidad física (Toledo, 2008). Mucho menos se puede creer que con leyes sociales como las económicas se puedan reducir a un solo tipo de análisis basado en la materialidad física o biológica, como lo hace la economía ambiental, reduciendo todos los análisis a valores económicos o monetarios (Sánchez, 2016). Por el contrario, se trata de tomar en cuenta las leyes físicas y las leyes biológicas en cada nivel de análisis para mantener la complejidad, y de esta manera respetar las limitaciones de cada una de las categorías.

\section{Metabolismo sociedad-naturaleza}

Karl Marx (1971) desde su texto "Elementos fundamentales para la crítica de la economía política” más conocido como "Los Grundrisse", esbozó que, para la satisfacción de las necesidades humanas es necesaria la transformación de la naturaleza en objetos cargados de subjetividad; lo cual requiere del trabajo humano. En el sistema económico actual, el trabajo, la materia, los objetos y la tecnología se encuentran en un conjunto de procesos de intercambio con un medio de comunicación económico que conecta a los diferentes actores dentro del mercado global, este conector es el dinero. El mercado, el dinero y el plusvalor que genera valor no se pueden concebir como fenómenos ontológicos existentes desde siempre, sino que son categorías en las cuales se fundamenta el capitalismo para su existencia histórica; las cuales tuvieron un inicio y tendrán un final.

Para Marx (2002) la historia del hombre es la narración temporal de la interacción metabólica de los seres humanos con la naturaleza, junto a su mediador: el trabajo. Por 
tanto, la historia humana en su totalidad es únicamente la narrativa del desarrollo o incremento de la complejidad entre ser humano y naturaleza, y todas las contradicciones sociales y ecológicas que esta conlleva.

La naturaleza no solo es determinada por la sociedad, sino que a su vez, la naturaleza produce una determinación a la sociedad, tanto de forma material como subjetiva. Todos los pueblos construyen un metabolismo a lo largo de su historia, ya que del trabajo está condicionado y a su vez es condicionante de los recursos disponibles a un medio, por lo que el metabolismo sociedad-naturaleza se configura como una huella de identidad distinta para cada sociedad. El sistema económico-social hegemónico de producción actual es el capitalismo, el cual ha dejado una huella ecológica profundamente marcada en el sistema natural (Marini, 1993); y designando a cada región en el mundo un papel dentro de su sistema lineal de funcionamiento entre extracción, producción, distribución, consumo y excreción (Toledo, 2008).

El análisis dialéctico del método propuesto por Breilh (2011) e interpretado en el Gráfico 3 , permite identificar una mutua modificación entre la materialidad social y natural. Ejemplo de esto es cómo la riqueza en recursos naturales o energéticos en los países periféricos determinan qué grado de inserción tendrán en la economía mundial; llegándose inclusive a fabricar conflictos bélicos o blandos en países periféricos, debido al agotamiento de los recursos naturales en los países centrales.

Esto se debe a que la esencia del capitalismo es un continuo crecimiento y crisis, puesto que el dinero tiene la capacidad de transformarse en capital e incrementar su valor por la explotación de los seres humanos (Marx, 2002) y de la naturaleza. No puede existir un crecimiento material infinito en un mundo de recursos finitos; mucho menos si la tierra pierde su capacidad de resiliencia frente a la mitigación de problemas graves (Daly y Townsend, 1993), como lo que está ocurriendo con el calentamiento global.

En América Latina el problema de "in" sustentabilidad es aún más grave, ya que los recursos naturales exportados aumentan cada vez más en cantidad y disminuyen en precio. En el caso del Ecuador se puede apreciar balances materiales y comercial negativos, ya que se exportan muchas más toneladas de materiales que las importadas, sin embargo el costo monetario de sus importaciones supera el de las exportaciones (Vallejo, 2006). Estas cifras podrían ser peores de agregarse los gastos incurridos por mitigación o curación de enfermedades, o los costos por remediación de pasivos ambientales; como por los daños del caso conocido como "Chevron/Texaco" (Serrano, 2013).

En el Figura 2, se puede observar la acentuación de la tendencia a tener balances negativos durante el acogimiento de las políticas conocidas como el "Consenso de Washington" en el balance comercial no petrolero para los años 1980 a 2004 
Figura 2

Saldo comercial y material no petrolero

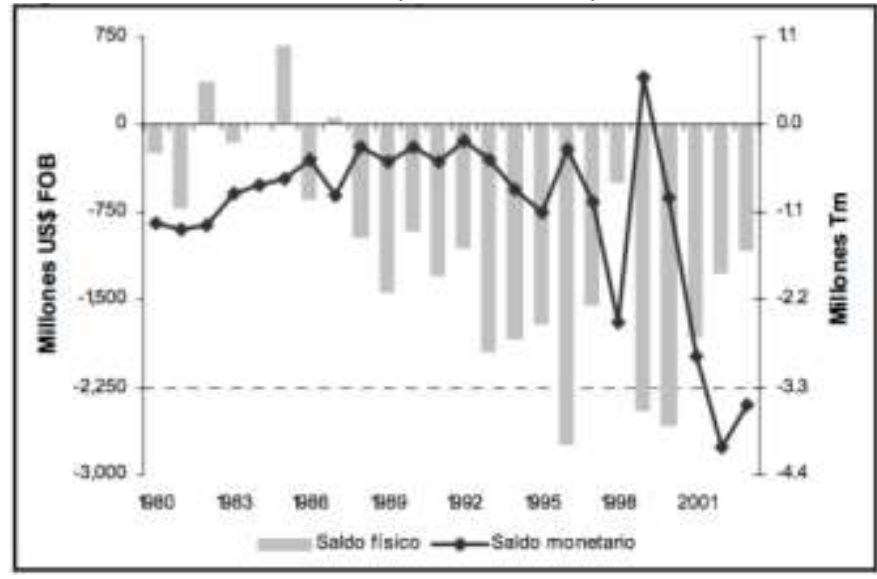

Fuente: Vallejo, 2006.

¿A qué se debe entonces que los países de América Latina estén "obligados" a mantener sus políticas extractivistas? Una respuesta desde la base material del método dialéctico se da en el hecho de que la región latinoamericana no produce conocimiento tecnológico ni desarrolla suficiente tecnología; sin embargo, usa de muy diversas formas los productos tecnológicos de países centrales. Esto fomenta una dependencia de productos elaborados en otras regiones, la cual debe ser compensado con otras formas de valor; como es la extracción de materias primas.

Como se pudo observar en el Gráfico 6, el trabajo de extracción de materias primas realizado en Ecuador es cada vez menos valorado, mientras que existe divergentemente un incremento del valor en el trabajo intelectual manejado desde monopolios $u$ oligopolios del conocimiento concentrados en países centrales (Negri, 1992). La ciencia y tecnología no son neutrales; por lo que, en el surgimiento de la era postindustrial existen nuevas formas de control de la producción como el monopolio cognitivo, que se identifica por su capacidad de extraer plusvalor a través de títulos de propiedad intelectual (Míguez et al. 2016).

El monopolio cognitivo incrementa las brechas de desigualdades tecnológicas y mercantiles entre el norte y el sur del planeta; haciendo que la posesión oligopólica del conocimiento tecnológico no permita la igualación de las tasas de productividad, con lo cual no existiría una mayor cuota de plusvalía para el norte del planeta. Este esquema es complementario a lo desarrollado por Emmanuel (1972) y Amin (1974), quienes explican cómo las naciones desfavorecidas deben ceder gratuitamente parte de su producción para disfrutar de beneficios en mercancías importadas y que no pueden producirse endógenamente; algo que Marini (1973) llamó "súper explotación”.

El comercio mundial facilita una división social del trabajo desagregada entre sus actores; por lo que se requiere de acuerdos que articulen a quienes les corresponderá la a) apropiación de los recursos desde la naturaleza; b) la transformación a productos elaborados; c) la distribución comercial del excedente producido, d) la asignación desigual del consumo, y e) quién recibe los residuos excretados a su ambiente (Toledo, 
2008). Por lo que, en un objeto sea imposible saber quién, cómo o donde se lo apropió, circuló, produjo, distribuyó, consumió y excretó (Marx, 2002).

Esto causa dos problemas, el primero es la asignación de América Latina en el proceso de apropiación de recursos naturales, que es una de las piezas en la máquina del sistema económico mundial, cuyos costos sociales y naturales no se visibilizan ni valoran en los bajos precios de sus mercancías. El segundo problema son los acuerdos con los que se logra la dependencia, los cuales son tanto institucionalizados, como por ejemplo los tratados de libre comercio, préstamos de bancos de desarrollo; y otros acuerdos que no requieren de ningún convenio explícito; lográndose el condicionamiento material previo al condicionamiento económico.

Una solución a esto propuesta por los gobiernos progresistas de la región es el fomento del desarrollo endógeno, visto como el proceso de industrialización (CELAC, 2013). Sin embargo, la evidencia indica que la industrialización endógena se puede realizar cuando los estados tienen grandes extensiones territoriales; algo que se realizaría si existiesen políticas reales de integración entre los países latinoamericanos (García 1959).

Contrario a esto, la región en los últimos años ha vivido un proceso de desindustrialización, ligado a un proceso de reprimarización de su economía (CEPAL, 2011). Esto se debe a qué, el crecimiento alcanzado en los últimos años situó fuertemente a América Latina como el proveedor de materias primas del nuevo actor en el escenario político como lo es China; quien a su vez elaboró productos industrializados que sustituyeron por menores precios a los fabricados en la región latinoamericana, desindustrializándola (Guajardo et al., 2016). En el Figura 3 se muestran las tasas de crecimiento del PIB chino y latinoamericano, donde se puede notar la correlación positiva entre el crecimiento y el decrecimiento en ambas regiones en los últimos años.

Figura 3

Porcentaje en el crecimiento del PIB entre China y América Latina

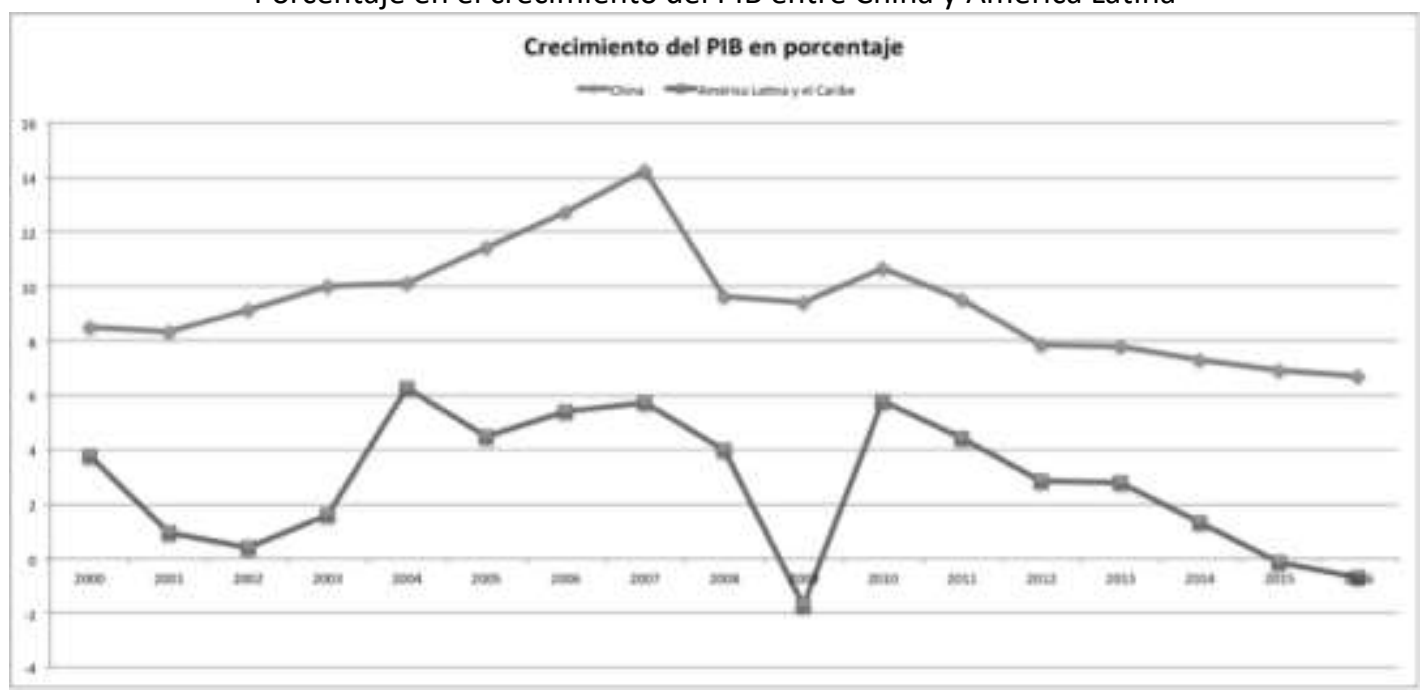

Fuente: Banco Mundial, 2017. 
La base material del método dialéctico propuesto por Marx no trata únicamente del entendimiento de la realidad, sino la toma de acción para modificarla (Marx, 2011). Sin embargo, una acción a nivel local solo tendrá un nivel de incidencia local; requiriéndose el escalamiento de las estrategias para producir un condicionamiento general en el sistema económico global. Así como para el caso de América Latina, existió un condicionamiento material extra regional previo al condicionamiento económico, desde América Latina puede existir un condicionamiento económico para lograr un cambio material.

Un primer condicionamiento transitorio desde la región puede ser la incorporación de los costos reales para la producción y reproducción de las materias primas, los cuales se basan en la conservación material de los servicios ecosistémicos (Toledo, 2008); cuya escasez pone en riesgo a todo el sistema económico globalizado. Un aumento en el precio de los recursos disminuirá la demanda, y exigirá el desarrollo de nuevas tecnologías menos intensivas en el uso de materiales y energía.

Esto ha sucedido con el petróleo en años recientes, donde el incremento del precio desaceleró su demanda hasta casi estabilizar el consumo en Europa y Estados Unidos (British Petroleum, 2017). Una tendencia mundial a la desmaterialización obligaría a América Latina a la inversión en nuevos componentes que diversifiquen su economía; y que a su vez ayuden a la conservación de la naturaleza, la mitigación del cambio climático, y a la disminución de la inequidad mediante el aumento de los puestos de trabajo.

\section{Discusión.}

Para poder lograr una ciencia que impulse a la acción, es necesario actuar en los conectores sociales que interactúan en la formación de la realidad; por tanto, para que la praxis transformadora se vuelva praxis normalizadora de procesos, se debe lograr la acción en la cotidianidad.

Las propuestas resultantes del presente trabajo no requieren acabar con la modernidad ni mucho menos con el sistema económico actual, ya que no existe un proyecto que abarque el nivel de complejidad que se requiere para un cambio total del sistema económico mundial. Tampoco se requiere la invención o desarrollo de nuevas tecnologías más limpias sin que se desarrollen paralelamente las fuerzas políticas necesarias para que este cambio se dé al nivel cotidiano.

El desarrollo de la comunicación y de una moneda única ha acelerado el comercio global, conectando las necesidades y las capacidades de la región con las de otras regiones, pero manteniéndose el monopolio de la tecnología en los países centrales, lo cual ha incrementado la explotación social y control de los recursos de la naturaleza en los países periféricos.

Por tanto, si bien resulta imposible un cambio del sistema económico actual, si resulta posible empezar a adquirir la fuerza necesaria para la modificación de un solo conector histórico que lo articule y que con el tiempo pueda condicionar en algo la destrucción de 
la naturaleza debido al incremento de consumo. Este conector puede ser la necesidad de valorar con herramientas de la economía ecológica los costos reales necesarios en la producción y reproducción de los recursos naturales debido a su creciente escasez, con lo cual se lograría un condicionamiento material.

\section{Conclusiones.}

- Desde la época de la colonia, el comercio mundial determinó a América Latina el papel exportador de materias primas; adquiriendo como categoría identificadora la apropiación de los recursos naturales. Esta tendencia no solo continúa en la actualidad, sino que se ha intensificado. La transformación hacia una sociedad postindustrial ha hecho que se intensifique la ley del valor, creándose una súper explotación de la sociedad y la naturaleza mediante la monopolización de la tecnología en los países centrales. Este "monopolio cognitivo" crea una baja en los precios de las materias primas, obteniéndose en los países del sur saldos materiales y económicos negativos.

- La razón por la cual se da este problema no es por la tendencia política de los gobernantes; sino porque América Latina está determinada al uso de las tecnologías creadas en los países centrales, las cuales no la produce internamente; requiriéndose por tanto de un compensador económico en el mercado para su intercambio, como es la extracción de las materias primas para tener mayor participación en el mercado mundial. Esto se evidenció en años recientes, en los cuales la región experimentó de un crecimiento económico con tendencias similares al crecimiento de China, pero recíprocamente las ventas de materiales elaborados a menor precio de este país redujeron la producción de las industrias internas, haciendo más rentable la exportación de materias primas frente a los bienes con mayor valor intelectual.

- Por tanto, es necesario adquirir fuerza para la modificación de los conectores históricos que articulan la economía mundial, y que con el tiempo puedan condicionarla. Estos conectores pueden surgir de la necesidad de mantener los recursos naturales considerando los costos reales causados en su producción y reproducción; para lo cual pueden ser útiles los métodos de la economía ecológica, las cuales toman en cuenta las limitaciones naturales en el desarrollo y crecimiento económico.

\section{Referencias bibliográficas.}

Amin, Samir (1974). Accumulation on a World Scale (Londres: Harvester Press).

Banco Mundial (2010). Recursos naturales pueden impulsar prosperidad a largo plazo de América Latina, según informe del Banco Mundial. Banco Mundial. Recuperado el 25 de septiembre de 2017. Recuperado desde: http://www.bancomundial.org/es/news/feature/2010/09/16/natural- 
resources-can-fuel-latin-americas-long-term-prosperity-argues-world-bankreport.

Breilh, Jaime (2011). Epidemiología critica. Ciencia emancipadora e intercultural. Editorial Lugar: Buenos Aires.

British Petroleum (2017). Statistical Review of World Energy. BP: Londres.

CELAC (2013). Desarrollo productivo e Industrialización en América Latina y el Caribe. Impreso en la Secretaría Permanente del SELA: Caracas.

CEPAL (2011, Noviembre 7). Reprimarización y desindustrialización en América Latina, dos caras de la misma moneda. En Segunda Mesa Redonda sobre Comercio y Desarrollo Sostenible. Recuperado desde: https://www.cepal.org/sites/default/files/events/ files/presentacion_sebastian_herreros_y_jose_duran.pdf

Cornejo, Rodrigo, Natalia Albornoz, Diego Palacios (2016). Subjetividad, realidad y discurso entre el determinismo estructuralista y el construccionismo social. Cinta moebio 56: 121-135. doi: 10.4067/S0717-554X2016000200001.

Dallanegra, Luis (2010). “Teoría y metodología de la geopolítica. Hacia una geopolítica de la "construcción de poder"". Revista mexicana de ciencias políticas y sociales, 52(210), 15-42. Recuperado en 15 de septiembre de 2017. Recuperado desde: http://www.scielo.org.mx/scielo.php?script=sci_arttext\&pid= S0185$19182010000300002 \& \operatorname{lng}=\mathrm{es} \& \operatorname{tlng}=\mathrm{es}$.

Daly, Herman, Kenneth Townsend (1993). Valuing the Earth: Economics, Ecology, Ethics. MIT Press: Cambridge.

Echeverría, Bolívar (1998). La modernidad de lo barroco. Ediciones ERA: México.

Emmanuel, Arghiri (1972). unequal Exchange. A Study of Imperialism of Trade (Londres: NLB).

Engels, Friedrich (1961). La Dialéctica de la Naturaleza.

Eysenck, Hanse (1971). Race, Intelligence, education. Temple Smith: Londres.

Field, Barry y Martha Field (2003). Economía ambiental. McGraw-Hill: Madrid.

Foucault, Michel (1966). Las palabras y las cosas: una arqueología de las ciencias humanas. Éditions Gallimard. París.

Gossen, Hermann (1881). Entwicklung der Gesetze des menschlichen Verkehrs und der daraus fliessenden Regeln für menschliches Handeln. Colonia. 
Guajardo, Jorge, Manolo Manuel, Dante Sica (2016). La industria latinoamericana ¿Y el rol de China cuál es? Atlantic Council: Washington, DC.

Guba, Egon (1990). The paradigm dialog. Newbery Park: Sage.

Haushofer, Karl (1986). De la géopolitique. Fayard: París.

Kant, Immanuel (2005). Crítica de la razón pura.

Kuznets, Simon (1955). "Economic Growth and Income Inequality" The American Economic Review, Vol. 45, No. 1, pp. 1-28. Pittsburgh.

Lewontin, Richard, Rose Steve, Leon Kamin (1984). No está en los genes. Crítica del racismo biológico. Grijalbo Mondadori: Barcelona.

Marini, Ruy (1973). Dialéctica de la dependencia. Siglo del hombre editores: Bogotá.

Marini, Ruy (1993). America Latina: democracia e integración, Nueva Sociedad, Caracas.

Martínez-Alier, Joan (1999). Introducción a la economía ecológica. Editorial RUBES: Barcelona.

Martínez-Alier, Joan (2005). El ecologismo de los pobres: conflictos ambientales y lenguajes de valoración. Icaria Editorial: Barcelona.

Marx, Karl (2011). Tesis sobre Feuerbach. En: Obras escogidas de K. Marx y F. Engels (Vol. 1, pp. 7-10). Moscú: Editorial Progreso.

Marx, Karl; Federico Engels (1974). La Ideología Alemana. Ediciones Pueblos Unidos, Montevideo.

Marx, Karl (1971). Elementos fundamentales para la crítica de la economía política. Siglo XXI Argentina Editores.

Marx, Karl (1980). Contribución a la Crítica de la Economía Política. Siglo XXI México Editores.

Marx, Karl (2002). El Capital tomo I. Siglo XXI México Editores.

Meadows, Donella, Gary Meadows, Jorgen Randers, William Behrens (1972). The Limits to Growth. Universe Books: New York.

Míguez, Pablo y Lima, Jacob (2016); "El trabajo cognitivo en el capitalismo contemporáneo" CENDES [en línea] 2016, 33 (Septiembre-Diciembre). Recuperado el 25 de septiembre de 2017] Disponible en:〈http://www.redalyc.org/articulo.oa?id=40352382005>

Negri, Antonio (1992). La teoría del valor trabajo: crisis y problemas de reconstrucción en la postmodernidad. Akal: Madrid. 
García, Plácido (1959). "Problemas de integración industrial Latinoamericana" Revista de Economía y Estadística, Tercera Época, Vol.3, No.1-2-3-4:1, 2, 3 y 4 Trimestre, pp.99-117. Córdova.

Rostow, Walt (1961). Las etapas del crecimiento económico. Fondo de Cultura Económica, México.

Sánchez, Renato (2016). Análisis de riesgos y salvaguardas ambientales para la actividad financiera del Banco del Sur: estudio de caso del recurso hídrico. Recuperado desde: http://repositorio.flacsoandes.edu.ec/ bitstream/10469/9767/2/TFLACSO-2016RGSP.pdf

Sánchez, Renato (2017). Salvaguardas ambientales para la banca de desarrollo regional desde la Economía Ecológica en Perspectivas de la economía ecológica en el nuevo siglo. Xochimilco.

Serrano, Helga (2013). Caso Chevron-Texaco cuando los pueblos toman la palabra. Universidad Andina Simón Bolívar. Quito.

Smith, Adam (1994). La riqueza de las naciones. Edición de Carlos Rodríguez Braun. Alianza Editorial Madrid.

Tansley, Arhur (1934); “Observatios on Tropical African Grasslands” Journal of Ecology 22: Londres.

Toledo, Victor (2008); "Metabolismos rurales: hacia una teoría económico-ecológica de la apropiación de la naturaleza" Revista Iberoamericana de Economía Ecológica. Vol. 7: 1-26.

Torres, Guillermo (2015); "El retorno de Karl Marx y la crítica de la economía ecológica la economía política ecológica” Pensamiento al margen n², 2015.

Vallejo, María (2006). La estructura biofísica de la economía ecuatoriana: el comercio exterior y los flujos ocultos del banano. Editorial Abya-Yala: Quito.

WIDER (2000). World Income Inequality Database. United Nations University. Helsinki.

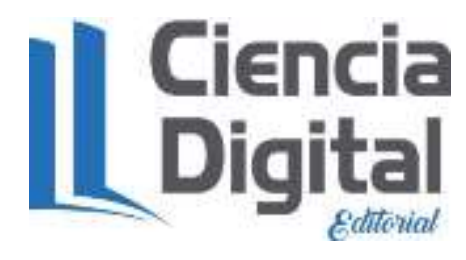




\section{PARA CITAR EL ARTÍCULO INDEXADO.}

Sánchez Proaño, R. (2021). Análisis de la primarización de la economía en América Latina desde la base material del método dialéctico . ConcienciaDigital, 4(3.2), 79-94. https://doi.org/10.33262/concienciadigital.v4i3.2.1852

\section{LCiencia}

El artículo que se publica es de exclusiva responsabilidad de los autores y no necesariamente reflejan el pensamiento de la Revista Conciencia Digital.

El artículo queda en propiedad de la revista y, por tanto, su publicación parcial y/o total en otro medio tiene que ser autorizado por el director de la Revista Conciencia Digital.

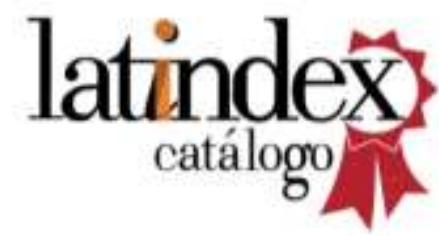

